\title{
Electroosmotic Flow of Salt-Free Power-Law Fluids in Micro/Nanochannels with Fluid Slip
}

\author{
S. H. Chang \\ Department of Mechanical Engineering, Far East University, Tainan, 74448, Taiwan \\ $\dagger$ †mail:shchang@mail.feu.edu.tw
}

(Received August 7, 2019; accepted December 8, 2019)

\begin{abstract}
Electroosmotic flow of salt-free power-law fluids through planar slit and cylindrical micro and nanochannels with fluid slip is theoretically analyzed. Analytical solutions are obtained to investigate the effects of flow behavior index, channel size, applied electric field strength, Gouy-Chapman length (or surface charge density), and fluid slip length on the velocity distribution and volumetric flow rate. The results show that the electroosmotic flow velocity and thereby the flow rate for shear-thinning fluids are many times larger than those for Newtonian and shear-thickening fluids for the ranges of applied electric field strength and surface charge density usually encountered in practice. Such augmentation can be further amplified by increasing the surface charge density, applied electric field strength and fluid slip. Furthermore, the electroosmotic flow velocity profile of shear-thinning fluids becomes more plug-like as the ratio of channel half-width (or radius) to GouyChapman length increases. However, such a profile for shear-thickening fluids always exhibits a parabolic-like flow pattern regardless of the ratios of channel half-width (or radius) to Gouy-Chapman length.
\end{abstract}

Keywords: Electroosmotic flow; Power-law fluid; Counterion-only; Fluid slip.

NOMENCLATURE

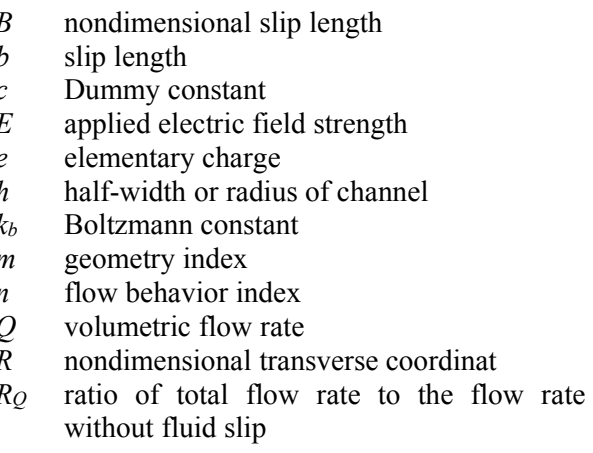

\section{INTRODUCTION}

Electroosmosis is one of the basic electrokinetic phenomena in which the ionized fluid flows through a stationary charged surface when an external electric field is applied. Owing to its numerous advantages such as ease of fabrication, no moving parts, plug-like velocity profile, high reliability, no noise and better flow control, electroosmotic transport has been widely used in chemical and biomedical analysis (Harrison et al. 1993; Manz et al. 1994), fuel cells (Eikerling et al. 2001), and

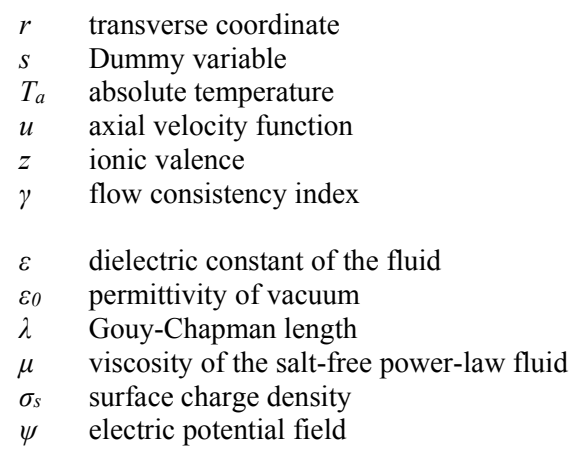

micro/nanofluidic devices (Laser and Santiago, 2004; Manz et al. 1994; Stone et al. 2004). Electroosmotic flow (EOF) of Newtonian fluids in micro and nanochannels has been well studied over the years (Burgreen and Nakache, 1964; Levine et al. 1975; Mala et al. 1997; Rice and Whitehead, 1965; Xuan and Li, 2005; Yan et al. 2007, 2009). However, many fluids, which are often analyzed in micro and nano-fluidic systems, such biofluids, polymer solutions and colloidal suspensions, are nonNewtonian in nature. This non-Newtonian effect on EOF was first investigated by Bello et al. (1994) who 
found that the experimentally measured electroosmotic velocity of a polymer (methyl cellulose) solution is much higher than that predicted with the classic Helmholtz-Smoluchowski velocity. About a decade later, Das and Chakraborty (2006) obtained the first analytical solution for EOF of power-law fluids in slit microchannels with DebyeHückel linear approximation. Since then, many theoretical (Berli and Olivares, 2008; Chang, 2016; Escandón et al. 2015; Vasu and De, 2010; Zhao and Yang, 2012; Zhao et al. 2008), numerical (Tan et al. 2009; Zimmerman et al. 2006), and experimental (Berli, 2010; Chang and Tsao, 2007; Olivares et al. 2009) studies have been reported on EOF of nonNewtonian fluids in micro and nano-channels. For more details about electroosmosis of non-Newtonian fluids, the reader is referred to the review articles (Zhao and Yang, 2013) and the references therein.

All the aforementioned studies are based on the noslip boundary condition. However, apparent fluid slip has been observed in recent experiments (Choi et al. 2003; Pit et al. 2000; Tretheway and Meinhart, 2002) at the micro/nano scales. The conventional silica channel surface used in micro and nano-fluidic devices is hydrophilic, while hydrophobic polymers such as poly (dimethylsiloxane) (PDMS) have become increasingly attractive in fabricating such devices (Duffy et al. 1998; Ocbvirk et al. 2000). The pioneering research of fluid slip effect on EOF of Newtonian fluids was probably done by Muller et al. (1986). Later, several works (Ajdari and Bocquet, 2006; Joly et al. 2004; Yang and Kwok, 2003) reported in the literature discussed the role of Newtonian fluid slip in the electrokinetic flow over hydrophobic surfaces. Recently, Misra and Sinha (2015) theoretically investigated the non-Newtonian and slip effects on EOF and heat transfer of a second grade fluid in hydrophobic microchannels. Each of these papers revealed a common feature that fluid slip could considerably enhance EOF velocity, thereby increasing the electroosmotic mobility and flow rate.

The working fluids in the above mentioned studies are limited to the electrolyte solutions containing both co-ions and counterions. However, salt-free solutions in which counterions are only present in the media are common in lamellar liquid crystals formed by ionic amphiphiles (Engström and Wennerström, 1978), and also when, for example, colloidal particles, clay sheets, surfactant micelles or bilayers whose surfaces contain ionizable groups interact in water (Israelachvili, 1991). Recently, the lowpermittivity organic solvents used in electronic paper (Chang et al. 2010) and the proton-water complexes in polymer electrolyte membranes (Eikerling et al. 2001) are also found to be salt-free media. Even an electrolyte solution with low salt concentration (< $10^{-5} \mathrm{M}$ ) can be treated as a salt-free solution (van der Heyden et al. 2006). An exact solution for $\mathrm{EOF}$ of a proton-water solution in a cylindrical nanochannel of polymer electrolyte membranes was presented by Berg and Ladipo (2009). Recent theoretical works for transient EOF of salt-free fluids in microchannels (Chang, 2009, 2010, 2012) have shown that the electroosmotic mobility is dependent on the microchannel size, which is completely different from that in an electrolyte solution. This result has been validated with EOF experiments (Chang et al. 2010). More recently, Bandopadhyay and Chakraborty (2013) investigated the effect of ionic size on electroosmotic transport of salt-free solution through nanochannels. Unfortunately, none of these studies address the non-Newtonian or fluid slip effects on EOF of salt-free fluids.

The objective of this paper is to present a theoretical description of the EOF of a salt-free power-law fluid in slit and cylindrical micro/nanochannels with fluid slip. For this purpose, the mathematical formulation of the problem and the resulting analytical expressions of the EOF velocity and volumetric flow rate are given in Section 2. Then, parametric studies are conducted to investigate the effects of flow behavior index, channel size, applied electric field strength, Gouy-Chapman length (or surface charge density), and fluid slip on the EOF velocity distribution and volumetric flow rate in Section 3. Finally, a summary of the main results is provided in Section 4.

\section{MATHEMATICAL MODELLING}

Consider an infinitely long planar slit of width $2 h$ or cylindrical capillary of radius $h$, uniformly charged with the surface charge density $\sigma_{s}$ and filled with a power-law fluid containing only counterions of the valence $-z$, as shown in Fig. 1. Then, for the planar slit $(m=0)$, the electric potential field $\psi(r)$ has been derived as (Chang, 2010)

$\psi(r)=\frac{k_{b} T_{a}}{z e} \operatorname{lnsec}{ }^{2}\left(\frac{c r}{h}\right)$

while for the cylindrical capillary $(m=1)$ (Chang, 2009)

$\psi(r)=-\frac{2 k_{b} T_{a}}{z e} \ln \left[1-\frac{h}{2 \lambda+h}\left(\frac{r}{h}\right)^{2}\right]$

where $e$ is the elementary charge, $k_{b}$ is the Boltzmann constants, $T_{a}$ is the absolute temperature and the constant $c$ in Eq. (1) can be determined by

$2 \mathrm{ctan} c=\frac{h e z \sigma_{s}}{\varepsilon \varepsilon_{0} k_{b} T_{a}}=\frac{2 h}{\lambda}$

Here, $\varepsilon$ is the dielectric constant of the fluid, $\varepsilon_{0}$ is the permittivity of the vacuum, and the Gouy-Chapman length

$\lambda=\frac{2 \varepsilon \varepsilon_{0} k_{b} T_{a}}{z e \sigma_{s}}$

defines a layer near the charged surface within which most counterions are localized (van der Heyden et al. 2006). Note that $\lambda$ is inversely proportional to the surface charge density $\sigma_{s}$ and play a similar role as the Debye length does in electrolyte solutions.

When a uniform external electric field $E$ is applied along the negative axial direction, the fluid starts to move due to electroosmosis. Also, no pressure gradient is applied and gravitational force is negligible. For an axisymmetric, steady, fully developed flow, only the axial velocity $u(r)$ exists and then the momentum equation can be simplified to 
$\frac{d}{d r}\left(r^{m} \mu \frac{d u}{d r}\right)+E \varepsilon \varepsilon_{0} \frac{d}{d r}\left(r^{m} \frac{d \psi}{d r}\right)=0$

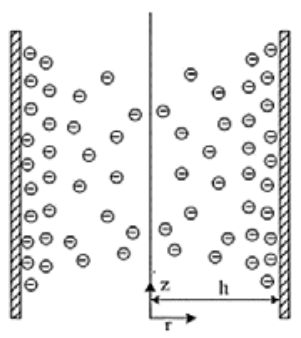

(a)

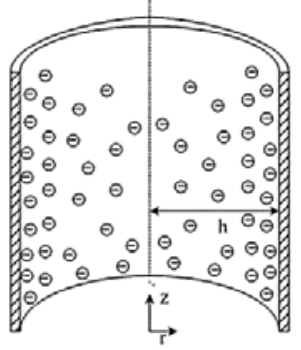

(b)
Fig. 1. Schematic diagrams of the symmetrically charged (a) planar slit of width $2 \mathrm{~h}$ and (b) cylindrical capillary of radius $h$, filled with a power-law fluid containing only mobile counterions.

where $\mu$ is the viscosity of a salt-free power-law fluid and given by (Chhabra and Richardson, 1999)

$\mu=\gamma\left(-\frac{d u}{d r}\right)^{n-1}$

Here, $\gamma$ is the flow consistency index and $n$ is the flow behavior index. Note that the power-law fluids are classified as the shear-thinning (pseudoplstic) fluids for $n<1$, Newtonian fluids for $n=1$, or the shearthickening (dilatant) fluids for $n>1$. Substitute Eq. (6) into Eq. (5) to give

$\frac{d}{d r}\left[r^{m} \gamma\left(-\frac{d u}{d r}\right)^{n}\right]-E \varepsilon \varepsilon_{0} \frac{d}{d r}\left(r^{m} \frac{d \psi}{d r}\right)=0$

which is subject to the symmetry at center $(r=0)$ and Navier's slip condition at wall $(r=h)$, i.e.

$\frac{d u}{d r}\left|r=0=0, u(h)=-b \frac{d u}{d r}\right|_{r=h}$

where $b$ is the slip length and positive. Note that the negative sign is chosen in Eqs. (6) and (8) because the velocity decreases with an increase in $r$ in both cases.

The exact solution to Eq. (7) with the boundary conditions Eq. (8) can be derived as

$u(r)=\int_{r}^{h}\left(\frac{E \varepsilon \varepsilon_{0}}{\gamma} \frac{d \psi}{d r}\right)^{\frac{1}{n}} d s-\left.b \frac{d u}{d r}\right|_{r=h}$

After substituting Eq. (1) into the above equation, we obtain the EOF velocity through the planar slit $(m=0)$

$$
\begin{aligned}
u(r) & =h\left(\frac{2 E \varepsilon \varepsilon_{0} k_{b} T_{a}}{\gamma z e \lambda}\right)^{\frac{1}{n}}\left\{\int_{R}^{1}\left[\frac{\tan (c s)}{\tan c}\right]^{\frac{1}{n}} d s+B\right\} \\
& =h\left(\frac{E \sigma_{s}}{\gamma}\right)^{\frac{1}{n}}\left\{\int_{R}^{1}\left[\frac{\tan (c s)}{\tan c}\right]^{\frac{1}{n}} d s+B\right\}
\end{aligned}
$$

where $R=r / h$ and $B=b / h$. Furthermore, the volumetric flow rate per unit length of the planar slit channel can be expressed as

$Q=2 h^{2}\left(\frac{\mathrm{E} \sigma_{s}}{\gamma}\right)^{\frac{1}{n}}\left\{\int_{0}^{1} \int_{R}^{1}\left[\frac{\tan (c s)}{\tan c}\right]^{\frac{1}{n}} d s d R+B\right\}$

where the first term represents the flow rate without fluid slip (i.e. $B=0$ ) and the ratio of total flow rate to this flow rate becomes

$$
R_{Q}=1+\frac{B}{\int_{0}^{1} \int_{R}^{1}\left[\frac{\tan (c s)}{\tan c}\right]^{\frac{1}{n}} d s d R}
$$

Similarly, the EOF velocity in a cylindrical capillary $(m=1)$ can be obtained from Eqs. (2) and (9) as

$$
\begin{aligned}
u(r) & =h\left(\frac{2 E \varepsilon \varepsilon_{0} k_{b} T_{a}}{\gamma z e \lambda}\right)^{\frac{1}{n}}\left\{\int_{R}^{1}\left[\frac{2 s}{2+\frac{h}{\lambda}\left(1-s^{2}\right)}\right]^{\frac{1}{n}} d s+B\right\} \\
& =h\left(\frac{E \sigma_{s}}{\gamma}\right)^{\frac{1}{n}}\left\{\int_{R}^{1}\left[\frac{2 s}{2+\frac{h}{\lambda}\left(1-s^{2}\right)}\right]^{\frac{1}{n}} d s+B\right\}
\end{aligned}
$$

and the volumetric flow rate is

$Q=2 \pi h^{3}\left(\frac{\mathrm{E} \sigma_{S}}{\gamma}\right)^{\frac{1}{n}}\left\{\int_{0}^{1} \int_{R}^{1}\left[\frac{2 s}{2+\frac{h}{\lambda}\left(1-s^{2}\right)}\right]^{\frac{1}{n}} d s R d R+\frac{B}{2}\right\}$

with corresponding ratio of the total flow rate to the flow rate without fluid slip

$$
R_{Q}=1+\frac{B}{2 \int_{0}^{1} \int_{R}^{1}\left[\frac{2 s}{2+\frac{h}{\lambda}\left(1-s^{2}\right)}\right]^{\frac{1}{n}} d s R d R}
$$

It is apparent from the above equations that the fluid slip B can enhance the EOF velocity and thereby the flow rate. Also, the EOF velocity and the flow rate are nonlinear with both parameters $h / \lambda$ and $E \sigma_{s} / \gamma$ due to the non-Newtonian fluid behavior. Moreover, the ratio of volumetric flow rate with fluid slip to that without fluid slip depends only on $n, B$, and $h / \lambda$, not on $E \sigma_{s} / \gamma$.

\section{RESULTS AND DISCUSSION}

The working fluid is assumed to be a power-law fluid containing only monovalent counterions $(z=1)$. Other parameters used in calculations are dielectric constant of medium $\varepsilon=24.3$ for ethanol (Chang et al. 2010), room temperature $T_{a}=298 \mathrm{~K}$, and flow consistency index $\gamma=10^{-2} \mathrm{~Pa} \mathrm{~s}^{\mathrm{n}}$ (Berli and Olivares, 2008). Hence, it follows from Eq. (4) that the relation between the surface charge density $\sigma s$ and the GouyChapman length $\lambda$ becomes $\lambda \sigma_{s} \fallingdotseq 1.105 \times 10^{-11}$ $\mathrm{Cm}^{-1}$. This implies that $\sigma_{s}=10^{-2} \mathrm{Cm}^{-2}$ yields $\lambda \fallingdotseq 1.1$ $\mathrm{nm}$. A direct numerical integration was applied to compute the EOF velocity and the flow rate using quad8.m in MATLAB and Simpson's rule with 500 grids.

Figure 2 shows the shape of the EOF velocity normalized with its velocity at the centerline of channels $u(0)$ for various salt-free power-law fluids through a planar slit channel without fluid slip at two different ratios of channel half-width (or radius) $h$ to Gouy-Chapman length $\lambda$. The corresponding results in a cylindrical capillary without fluid slip are shown in Fig. 3. For shear-thickening fluids $(n>1)$, the velocity profile always exhibits a parabolic-like flow 

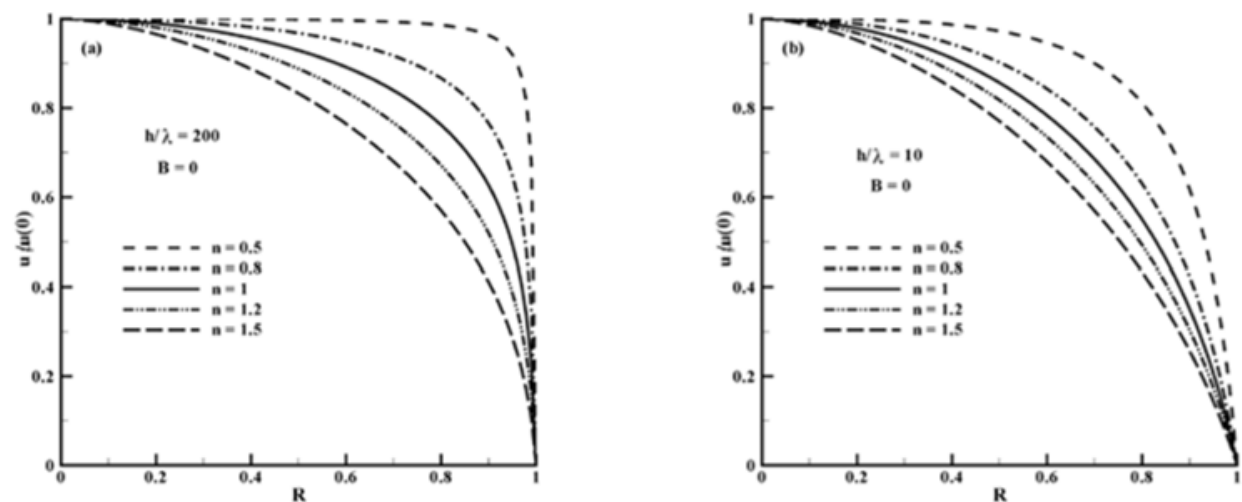

Fig. 2. Spatial profile of the normalized EOF velocity for various salt-free power-law fluids through a planar slit without fluid slip at (a) $h / \lambda=200$ and (b) $h / \lambda=10$. The reference velocity $u(0)$ is the EOF velocity at the centerline of channels.
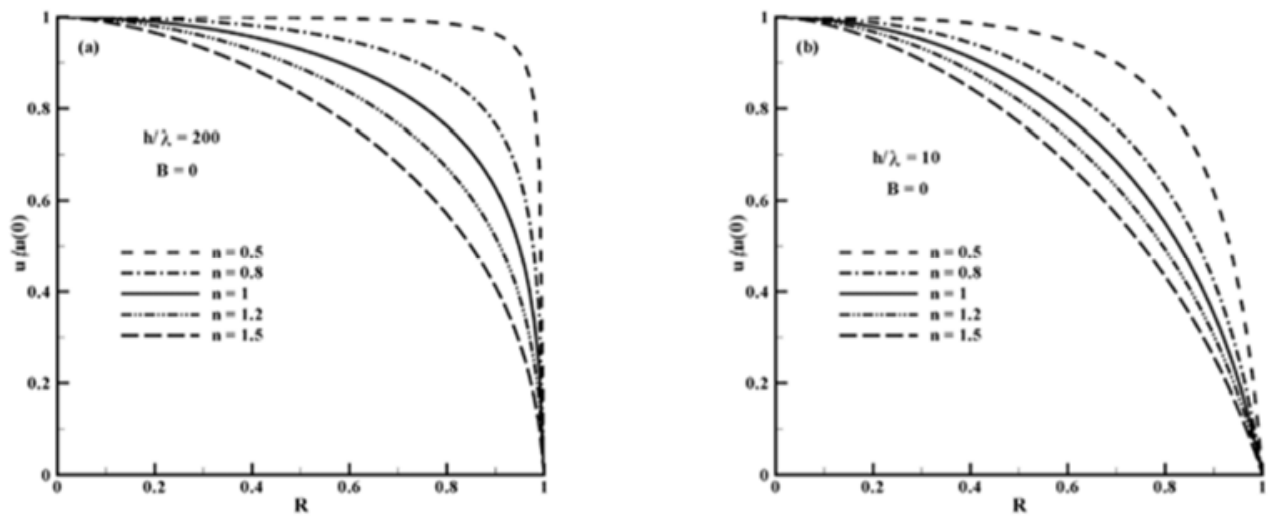

Fig. 3. Spatial profile of the normalized EOF velocity for various salt-free power-law fluids through a cylindrical capillary without fluid slip at (a) $h / \lambda=200$ and (b) $h / \lambda=10$. The reference velocity $u(0)$ is the EOF velocity at the centerline of channels.

pattern regardless of the values of $h / \lambda$. For shearthinning fluids $(n<1)$, however, such a profile becomes more plug-like as $h / \lambda$ increases. This is because the viscosity of shear-thinning fluids decreases significantly near the channel wall with increasing the value of $h / \lambda$. It should be pointed out that the fluid slip $B$ and the parameter $E \sigma_{s} / \gamma$ have no effect on the EOF velocity profile as can be seen from Eqs. (10) and (13).

Figure 4 shows the EOF velocity distributions of various salt-free power-law fluids inside the planar slit channels and cylindrical capillaries without fluid slip for different combinations of the parameters $h / \lambda$ and $E \sigma_{s} / \gamma$ while keeping $h=50 \mu \mathrm{m}$. It is clearly seen that the EOF velocity in both geometries increases as $E \sigma_{s} / \gamma$ increases or $h / \lambda$ decreases. Also, the EOF velocity in a planar slit channel is always larger than that in a cylindrical capillary provided that the capillary diameter equals to the planar slit width. Nevertheless, such a difference tends to disappear with a decrease in $h / \lambda$ (see Figs. 4(c) and (d)). This is due to the fact that their EOF velocities given by Eqs. (10) and (13) will approach to the same value as $h / \lambda \rightarrow 0$. Another notable result in Fig. 4 is that the velocity is larger (smaller) for pseudoplastic fluids $(n<1)$ than that for dilatant fluids $(n>1)$ when $E \sigma_{s} / \gamma$ is greater (less) than some critical value. Besides, the above critical value of $E \sigma_{s} / \gamma$ will reach 1 as $h / \lambda \rightarrow 0$ and increase with increasing $h / \lambda$. These interesting features stem from the competition between two opposite effects: As the flow behavior index $n$ is raised, the term $\left(E \sigma_{s} / \gamma\right)^{1 / n}$ in Eqs. (10) and (13) increases for $E \sigma_{s} / \gamma<1$ but decreases for $E \sigma_{s} / \gamma>1$, while the integral appearing in the same equations increases for all values of $h / \lambda$. As a result, at higher values of $E \sigma_{s} / \gamma$, which is enough to counterbalance the effect by a given value of $h / \lambda$, an increase in flow behavior index $n$ leads to a reduction in the EOF velocity; however, the opposite is true at low $E \sigma_{s} / \gamma$.

Since the general behavior of the EOF velocity in both geometries is similar, for brevity, only the results of volumetric flow rates through the cylindrical capillary are presented below. The variation of normalized volumetric flow rate in the cylindrical capillary as a function of the flow behavior index $n$ for various values of $E \sigma_{s} / \gamma$ while 

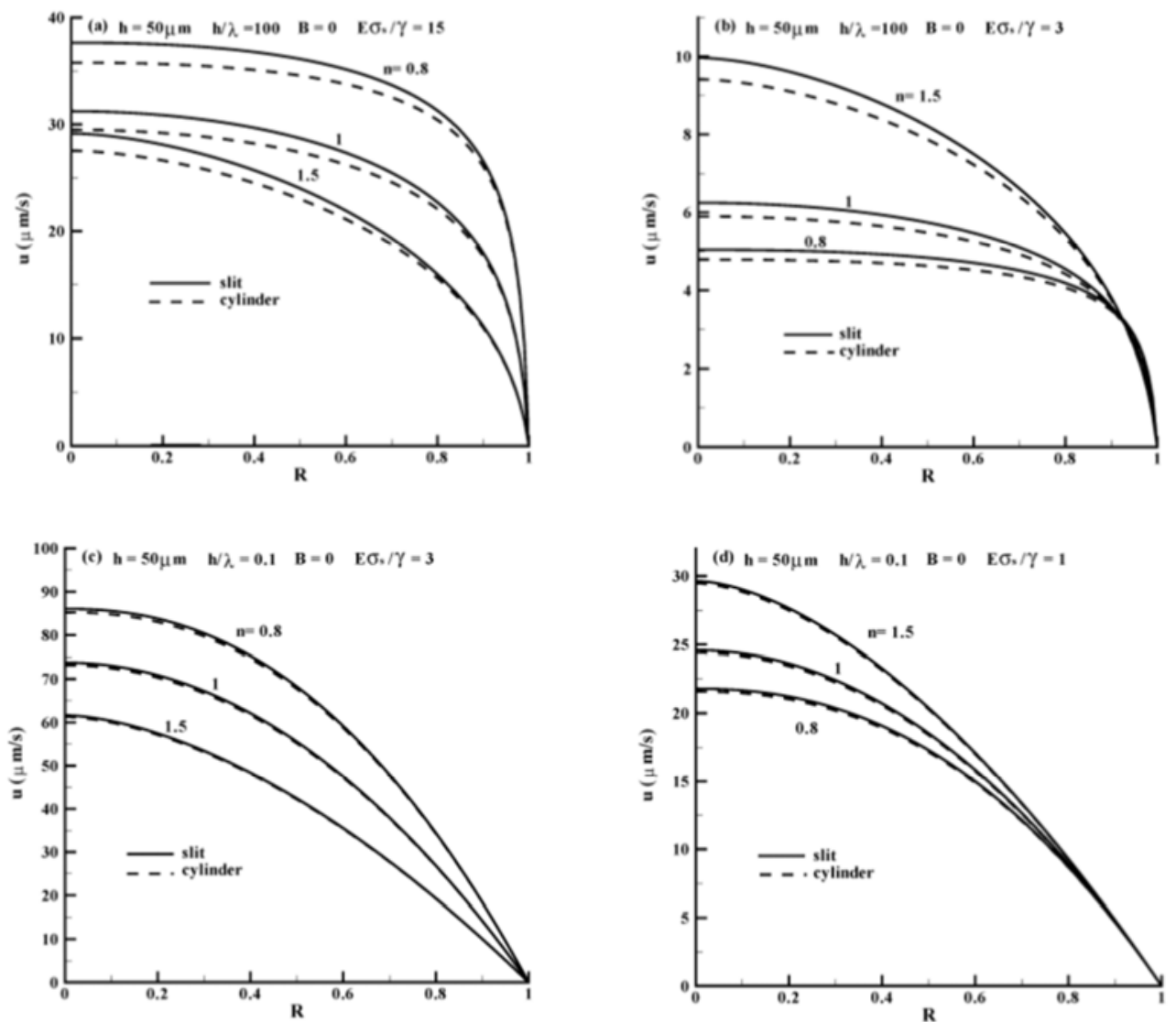

Fig. 4. Spatial distribution of EOF velocity for various salt-free power-law fluids through planar slit (solid lines) and cylindrical (dashed lines) micro/nanochannels without fluid slip at (a) $h / \lambda=100$,

$E \sigma_{s} / \gamma=15 \mathrm{~s}^{-\mathrm{n}}$ (b) $h / \lambda=100, E \sigma_{s} / \gamma=3 \mathrm{~s}^{-\mathrm{n}}(\mathrm{c}) h / \lambda=0.1, E \sigma_{s} / \gamma=3 \mathrm{~s}^{-\mathrm{n}}$ and (d) $h / \lambda=0.1, E \sigma_{\mathrm{s}} / \gamma=1 \mathrm{~s}^{-\mathrm{n}}$ with a fixed channel half-width (radius) $h=50 \mu \mathrm{m}$.
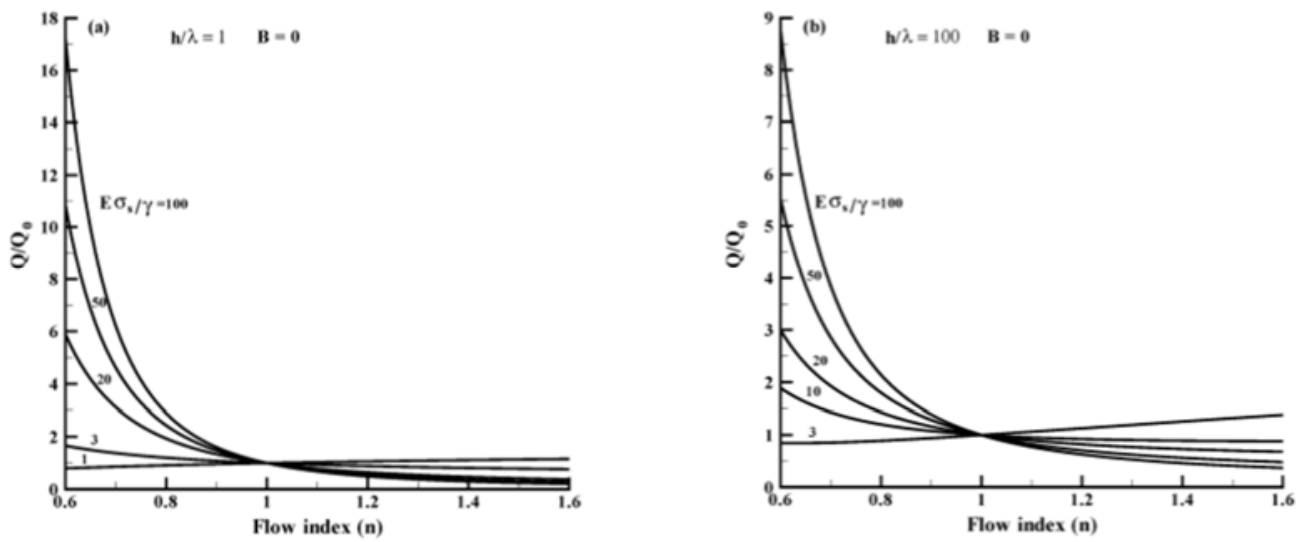

Fig. 5. Variation of the normalized volumetric flow rate for various salt-free power-law fluids through a cylindrical capillary without fluid slip at different values of $E \sigma s / \gamma$ with(a) $h / \lambda=1$ and $(b) h / \lambda=100$.

The reference flow rate $Q_{0}$ is the flow rate of Newtonian fluids $(n=1)$ without fluid slip $(B=0)$.

keeping $h / \lambda=1$ and 100 is displayed in Figs. 5(a) and (b), respectively, without fluid slip $(B=0)$. The reference flow rate $Q_{0}$ is that of Newtonian fluids $(n=1)$ without fluid slip $(B=0)$. At higher $E \sigma_{s} / \gamma$, which is common in practice, the flow rate is expected to decrease rapidly with the flow behavior index at low $n(n<1)$ and then tend to remain more or less unchanged at higher $n(n \geq 1)$. Under this condition, the volumetric flow rate of shear-thinning fluids $(n<1)$ is several times that of Newtonian and 

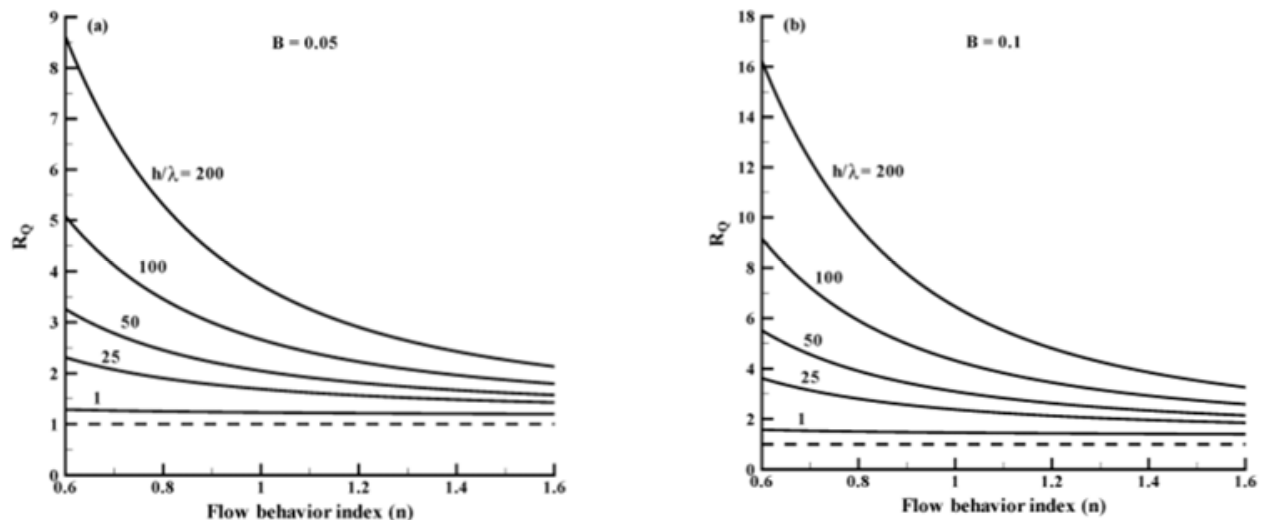

Fig. 6. Ratio $R_{Q}$ of the flow rate with fluid slip (a) $B=0.05$ and (b) $B=0.1$ to that without fluid slip ( $B$ $=0$ ) in a cylindrical capillary as a function of the flow behavior index $n$ for various values of $h / \lambda$. The dashed line $R_{Q}=1$ represents the case without fluid slip $(B=0)$.

shear-thickening fluids $(n \geq 1)$. This enhancement can be greatly enlarged by increasing the value of $E \sigma_{s} / \gamma$. Similar results have been reported in electrolyte power-law fluids by Zhao et al. (2008) and by Vasu and De (2010). At lower $E \sigma_{s} / \gamma$, the flow rate increases as $\mathrm{n}$ increases; however, such an increase is limited. Unlike the shear-thinning fluids, it is practically difficult to increase the volumetric flow rate of shear-thickening fluids. This implies that the shear-thinning fluid is more suitable to be the working fluid than the shear-thickening fluid in electroosmotic pumping.

Figure 6 shows the ratio $R_{Q}$ of the flow rate with fluid $\operatorname{slip}(B=0.05$ and 0.1$)$ to that without fluid slip $(B=0)$ in a cylindrical capillary as a function of the flow behavior index $\mathrm{n}$ for various values of $h / \lambda$. For a comparison, the result $R_{Q}=1$ for the case with $B=0$ is plotted as the dashed line in the figure. As expected, the flow rate is enhanced by increasing the fluid slip, where the largest enhancements are obtained in the region of small $n$ values in which the fluids exhibit the shear-thinning behavior. Such augmentation can be amplified up to about 9 (16) times with a 5 (10) \% fluid slip and even more when $h / \lambda>200$. Note that a $5 \%$ fluid $\operatorname{sip}(B=0.05)$ is easily achieved since a fluid slip of $B=0.07$ (Tretheway and Meinhart, 2002) has been experimentally measured in octadecyltrichlorosilane (OTS) coated microchannels.

\section{CONCLUSIONS}

A theoretical study on the electroosmotic flow of a salt-free power-law fluid through the planar slit and cylindrical micro/nanochannels with fluid slip has been presented in this paper. The exact analytical solutions for the EOF velocity are obtained by solving nonlinear Poisson-Boltzmann equation and Cauchy momentum equation. Based on the closedform solutions, the effects of flow behavior index, channel size, applied electric field strength, GouyChapman length (or surface charge density), and fluid slip on the velocity distribution and volumetric flow rate are discussed. The results show that the
EOF velocity profile of shear-thinning fluids $(n<1)$ becomes more plug-like as the ratio of channel halfwidth (or radius) to Gouy-Chapman length increases. However, such a profile for shear-thickening fluids $(n>1)$ always exhibits a parabolic-like flow pattern regardless of the ratios of channel half-width (or radius) to Gouy-Chapman length. Furthermore, the EOF velocity and thereby the flow rate for shearthinning fluids are many times larger than those for Newtonian and shear-thickening fluids for the ranges of applied electric field strength and surface charge density usually encountered in practice. Such augmentation can be further amplified by increasing the applied electric field strength, surface charge density and fluid slip.

\section{ACKNOWLEDGEMENTS}

This work was supported by the Ministry of Science and Technology, Taiwan, R.O.C under Grant No. MOST 104-2221-E-269 -008 and MOST 105-2221E-269 -003.

\section{REFERENCES}

Ajdari, A. and L. Bocquet (2006). Giant amplification of interfacially driven transport by hydrodynamic slip: Diffusio-osmosis and beyond. Physical Review Letters 96, 186102.

Bandopadhyay, A. and S. Chakraborty (2013). Ionic size dependent electroosmosis in ion-selective microchannels and nanochannels. Electrophoresis 34, 2193-2198.

Bello, M. S., P. De Besi, R. Rezzonico, P. G. Righetti and E. Casiraghi (1994). Electroosmosis of polymer solutions in fused silica capillaries. Electrophoresis 15, 623-626.

Berg, P. and K. Ladipo (2009). Exact solution of an electro-osmotic fllow problem in a cylindrical channel of polymer electrolyte membranes. Proceedings of the Royal Society A: Mathematical, Physical and Engineering 
Sciences 465, 2663-2679.

Berli, C. L. A. (2010). Output pressure and efficiency of electrokinetic pumping of non-Newtonian fluids. Microfluidics and Nanofluidics 8, 197207.

Berli, C. L. A. and M. L. Olivares (2008). Electrokinetic flow of non-Newtonian fluids in microchannels. Journal of Colloid and Interface Science 320, 582-589.

Burgreen, D. and F. R. Nakache (1964). Electrokinetic flow in ultrafine capillary slits. The Journal of Physical Chemistry 68, 10841091.

Chang, F. M. and H. K. Tsao (2007). Drag reduction in electro-osmosis of polymer solutions. Applied Physics Letters 90, 194105.

Chang, F. M. and Y. W. Chang, Y. J. Sheng and H. K. Tsao (2010). Size-dependent electro-osmosis in a microchannel with low-permittivity, saltfree media. Applied Physics Letters 97, 164101.

Chang, S. H. (2009). Transient electroosmotic flow in cylindrical microcapillaries containing saltfree medium. Biomicrofluidics 3, 012802.

Chang, S. H. (2010). Electroosmotic flow in slit microchannel containing salt-free solution. European Journal of Mechanics B/Fluids 29, 337-341.

Chang, S. H. (2012). Electroosmotic flow in a dissimilarly charged slit microchannel containing salt-free solution. European Journal of Mechanics B/Fluids 34, 85-90.

Chang, S. H. (2016). Comments on "Transient electroosmotic flow of Maxwell fluids with asymmetric zeta potentials through slit microchannel" [Eur. J. Mech. B Fluids 53 (2015) 180-189]. European Journal of Mechanics B/Fluids 58, 95-97.

Chhabra, R. P. and J. F. Richardson (1999). NonNewtonian Flow in Process Industries: Fundamentals and Engineering Applications. Butterworth-Heinemann, Oxford.

Choi, C. H., K. J. A. Westin and K. S. Breuer (2003). Apparent slip flows in hydrophilic and hydrophobic microchannels. Physics of Fluids 15, 2897-2902.

Das, S. and S. Chakraborty (2006). Analytical solutions for velocity, temperature and concentration distribution in electroosmotic microchannel flows of a non-Newtonian biofluid. Analytica Chimica Acta 559, 15-24.

Duffy, D. C., J. C. Mcdonald, O. J. A. Schueller and G. M. Whitesides (1998). Rapid prototyping of microfluidic systems in poly (dimethylsiloxane). Analytical Chemistry 70, 4974-4984.

Eikerling, M., A. A. Kornyshev, A. M. Kuznetsov, J. Ulstrup and S. Walbran (2001). Mechanisms of proton conductance in polymer electrolyte membranes. The Journal of Physical Chemistry $B$ 105, 3646-3662.

Engström, S. and H. Wennerström (1978). Ioncondensation on planar surfaces. A solution of the Poisson-Boltzmann equation for two parallel charged plates. The Journal of Physical Chemistry 82, 2711-2714.

Escandón, J., E. Jiménez, C. Hernández, O., Bautista and F. Méndez (2015). Transient electroosmotic flow of Maxwell fluids with asymmetric zeta potentials through slit microchannel. European Journal of Mechanics B/Fluids 53, 180-189.

Harrison, D. J., K. Fluri, K. Seiler, Z. H. Fan, C. S. Effenhauser and A. Manz (1993). Micromachining a miniaturized capillary electrophoresis-based chemical analysis system on a chip. Science 261, 895-897.

Israelachvili, J. N. (1991). Intermolecular and Surface Forces. Academic Press, San Diego, CA.

Joly, L., C. Ybert, E. Trizac and L. Bocquet (2004). Hydrodynamics within the electric double layer on slipping surfaces. Physical Review Letters 93,257805 .

Laser, D. J. and J. G. Santiago (2004). A review of micropumps. Journal of Micromechanics and Microengineering 14, R35-R64.

Levine, S., J. R. Marriott, G. Neale and N. Epstein (1975). Theory of electrokinetic flow in fine cylindrical capillaries at high zeta potential. Journal of Colloid and Interface Science 52, 136-149.

Mala, G. M., D. Li and J. D. Dale (1997). Heat transfer and fluid flow in microchannels. International Journal of Heat and Mass Transfer 40, 3079-3088.

Manz, A., C. S. Effenhauser, N. Burggraf, D. J. Harrison, K. Seiler and K. Fluri (1994). Electroosmotic pumping and electrophoretic separations for miniaturized chemical analysis system. Journal of Micromechanics and Microengineering 4, 257-265.

Misra, J. C. and A. Sinha (2015). Electro-osmotic flow and heat transfer of a non-Newtonian fluid in a hydrophobic microchannel with Navier slip. Journal of Hydrodynamics 27, 647-657.

Muller, V. M., I. P. Sergeeva, V. D. Sobolev and N. V. Churaev (1986). Boundary effects in the theory of electrokinetic phenomena. Colloid Journal of the USSR 48, 606-614.

Ocbvirk, G., M. Munroe, T. Tang, R. Oleschuk, K. Westra and D. J. Harrison (2000). Electrokinetic control of fluid flow in native poly (dimethylsiloxane) capillary electrophoresis devices. Electrophoresis 21, 107-115.

Olivares, M. L., L. Vera-Candioti and C. L. A. Berli (2009). The EOF of polymer solutions. Electrophoresis 30, 921-929. 
S. H. Chang / JAFM, Vol. 13, No. 4, pp. 1149-1156, 2020.

Pit, R., H. Hervet and L. Léger (2000). Direct experimental evidence of slip in hexadecane: Solid interfaces. Physical Review Letters 85, 980-983.

Rice, C. L. and R. Whitehead (1965). Electrokinetic flow in narrow cylindrical capillary. The Journal of Physical Chemistry 69, 4017-4024.

Stone, H. A., A. D. Stroock and A. Ajdari (2004) Engineering flows in small devices: Microfluidics toward a lab-on-a-chip. Annual Review of Fluid Mechanics 36, 381-411.

Tan, G. H., X. F. Li, Y. L. He and W. Q. Tao (2009) Electroosmotic flow of non-Newtonian fluids in microchannels. Journal of Non-Newtonian Fluid Mechanics 157, 133-137.

Tretheway, D. C. and C. D. Meinhart (2002). Apparent fluid slip at hydrophobic microchannel walls. Physics of Fluids 14, L9L12.

van der Heyden, F. H. J., D. J. Bonthuis, D. Stein, C. Meyer and C. Dekker (2006). Electrokinetic energy conversion efficiency in nanofluidic channels. Nano Letters 6, 2232-2237.

Vasu, N. and S. De (2010). Electroosmotic flow of power-law fluids at high zeta potentials. Colloids and Surfaces A: Physicochemical and Engineering Aspects 368, 44-52.

Xuan, X. and D. Li (2005). Electroosmotic flow in microchannels with arbitrary geometry and arbitrary distribution of wall charge. Journal of Colloid and Interface Science 289, 291-303.
Yan, D. G., C. Yang and X. Y. Huang (2007). Effect of finite reservoir size on electroosmotic flow in microchannels. Microfluidics and Nanofluidics 3, 333-340.

Yan, D. G., C. Yang, L. M. Miao, Y. C. Lam and X. Y. Huang (2009). Enhancement of electrokinetically driven microfluidic T-mixer using frequency modulated electric field and channel geometry effects. Electrophoresis 30, 3144-3152.

Yang, J. and D. Y. Kwok (2003). Effect of liquid slip in electrokinetic parallel-plate microchannel flow. Journal of Colloid and Interface Science 260, 225-233.

Zhao, C. and C. Yang (2012). Electroosmotic flows of non-Newtonian power-law fluids in a cylindrical microchannel. Electrophoresis 34, 662-667.

Zhao, C. and C. Yang (2013). Electrokinetics of nonNewtonian fluids: A review. Advances in Colloid and Interface Science 201-202, 94-108.

Zhao, C., E. Zholkovskij, J. H. Masliyah and C. Yang (2008). Analysis of electroosmotic flow of power-law fluids in a slit microchannel. Journal of Colloid and Interface Science 326, 503-510.

Zimmerman, W. B., J. M. Rees and T. J. Craven (2006). Rheometry of non-Newtonian electrokinetic flow in a microchannel $\mathrm{T}$ junction. Microfluidics and Nanofluidics 2, 481492. 